\title{
ADIÇÃO DE ANILINAS À NAFTOQUINONA EM ÁGUA E EM FASE SÓLIDA
}

\author{
Sabrina T. Martinez*, Bárbara V. Silva e Angelo C. Pinto \\ Instituto de Química, Universidade Federal do Rio de Janeiro, CT, Bl. A, Cidade Universitária, Ilha do Fundão, 21941-909 \\ Rio de Janeiro - RJ, Brasil \\ Vitor F. Ferreira e Fernando de Carvalho da Silva \\ Instituto de Química, Universidade Federal Fluminense, Outeiro de S. João Batista, s/n, 24210-150 Niterói - RJ, Brasil \\ Recebido em 16/7/11; aceito em 30/9/11; publicado na web em 4/1/12
}

\begin{abstract}
ADDITION OF AMINES TO NAPHTHOQUINONE IN WATER AND SOLID PHASE. Considering all the aspects of the principles of green chemistry, we present herein the addition reactions of amines to 1,4-naphthoquinone in water as solvent and also in solid phase. These reactions resulted in very colorful products that were easily monitored by thin layer chromatography and consequently easy to separate. Therefore, they are interesting experiments for experimental organic chemistry in the classrooms or in the laboratories.
\end{abstract}

Keywords: naphthoquinone; anilines; green chemistry.

\section{INTRODUÇÃO}

Substâncias contendo o núcleo quinona estão presentes em muitos produtos naturais, possuem um papel vital nos processos biológicos oxidativos, ${ }^{1}$ e são de considerável importância na química medicinal, pois estão frequentemente associadas a várias atividades biológicas, como anticancerígena, ${ }^{2}$ bactericida, ${ }^{3}$ antimalárica, ${ }^{4}$ tripanocida, ${ }^{5}$ tuberculostática $^{6}$ e fungicida. ${ }^{7}$

A introdução de um grupo vicinal oxidável, como aminas substituídas, ao núcleo quinônico pode influenciar as propriedades redox do núcleo 2-amino-1,4-naftoquinona, e a literatura mostra que o esqueleto aminoquinona está presente em diversos derivados bioativos. ${ }^{8}$

A introdução de um grupo amino à posição C-2 da 1,4-naftoquinona envolve, usualmente, a adição de um grupo nitro, nitroso ou azida $^{9}$ com posterior redução desses grupos com hidretos metálicos ou reações de substituição nucleofílica de grupos metoxila ${ }^{10}$ ou de átomos de halogênio no C-2. ${ }^{11} \mathrm{O}$ acoplamento oxidativo de alquil ou arilaminas à 1,4-naftoquinona ocorre em uma única etapa, é simples, rápido e de fácil execução.

Em trabalhos anteriores, nosso grupo de pesquisa desenvolveu um protocolo empregando ultrassom para a preparação de 2-aminocarboidratos-1,4-naftoquinonas em bons rendimentos e em curto tempo de reação. ${ }^{12}$ Recentemente, Cunha e colaboradores mostraram que a adição direta de benzilamina à 1,4-naftoquinona, em dimetilformamida, a temperatura ambiente, ocorre em rendimento moderado. ${ }^{13}$ Valderrama e colaboradores empregaram ácido de Lewis como catalisador $\left(\mathrm{CeCl}_{3} .7 \mathrm{H}_{2} \mathrm{O}, \mathrm{InBr}_{3}, \mathrm{FeCl}_{3}\right)$ para obtenção de aminoisoquinolinas em rendimentos que variaram de acordo com a amina utilizada. ${ }^{14}$

A água por ser o solvente universal, não ser inflamável e inofensiva ao meio ambiente tem muitas vantagens sobre os demais solventes orgânicos. Entretanto, a sua diferença de polaridade em relação aos reagentes orgânicos apolares a torna menos utilizada em reações químicas, devido à menor solubilidade de muitos compostos orgânicos em água. Contudo, nos últimos anos o uso da água como solvente em reações orgânicas vem aumentado consideravelmente. ${ }^{15}$

Entre as muitas classes de reações que podem ser feitas usando como solvente a água, pode-se destacar as reações de adição nucleofílica do tipo Michael. ${ }^{16}$

\footnotetext{
*e-mail: sabrinatmartinez@yahoo.com.br
}

Nos cursos de Química e de áreas afins é cada vez mais importante conscientizar os estudantes da necessidade de se desenvolver processos químicos que não agridam ao meio ambiente e satisfaçam os princípios do que se convencionou chamar de Química Verde ou Química Limpa. ${ }^{17}$ Isto obriga os professores de Química responsáveis por disciplinas experimentais a reverem muitas de suas práticas de ensino.

Dos vários princípios da Química Verde, alguns tocam mais de perto a Academia e devem ser praticados no dia a dia de laboratório. Por exemplo, deve-se sempre evitar a formação de resíduos. Quanto menos resíduos químicos forem formados, mais limpo é o processo. Por isso, o conceito de Economia Atômica deve ser ensinado desde o Ensino Médio. Isto significa que o ideal é que todos os átomos dos reagentes sejam incorporados aos produtos. E não menos importante é o uso de solventes atóxicos, baratos e inofensivos ao meio ambiente.

Outro aspecto interessante no desenvolvimento de experimentos para cursos de graduação é o acompanhamento visual da reação química, desde a mudança de cor do meio de reação ao acompanhamento da formação de produtos coloridos através da cromatografia em camada delgada (CCD).

Em continuação aos nossos estudos visando a elaboração de experimentos para cursos de graduação em Química e de áreas afins, ${ }^{18}$ descrevemos neste artigo um protocolo simples, eficiente, suave e ambientalmente amigável para a síntese de 2-(fenil)-amino-1,4-naftoquinonas através da reação de adição de anilinas à 1,4-naftoquinonas usando água como solvente, sem o uso de catalisadores de transferência de fase ou em fase sólida (sem solvente). Tal metodologia está em concordância com vários dos princípios da Química Verde, como a não geração de resíduos, economia atômica e o uso de solvente atóxico para a saúde humana e para o meio ambiente.

\section{RESULTADOS E DISCUSSÃO}

Garden e colaboradores ${ }^{19}$ descreveram recentemente a adição oxidativa de anilinas à 1,4-naftoquinona, gerando $\mathrm{N}$-aril-2-amino1,4-naftoquinonas, catalisada por acetato de cobre (II). Os autores estabeleceram que a ausência do catalisador de ácido de Lewis torna a reação mais lenta e resulta em baixos rendimentos, com a formação de muitos produtos colaterais.

Neste trabalho, a 1,4-naftoquinona reagiu com a anilina, $p$-metil-anilina e $p$-metóxi-anilina, em solução aquosa, conduzindo à formação 
imediata dos produtos de coloração bastante intensa, e em rendimentos que variaram de bons a moderados, indicando que a água, além de solvente, funciona bem como catalisador ácido (Esquema 1 e Figura 1).

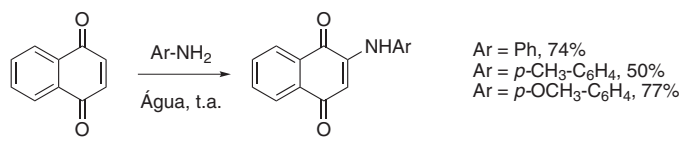

Esquema 1. Síntese de 2-(aril)-amino-1,4-naftoquinonas em água

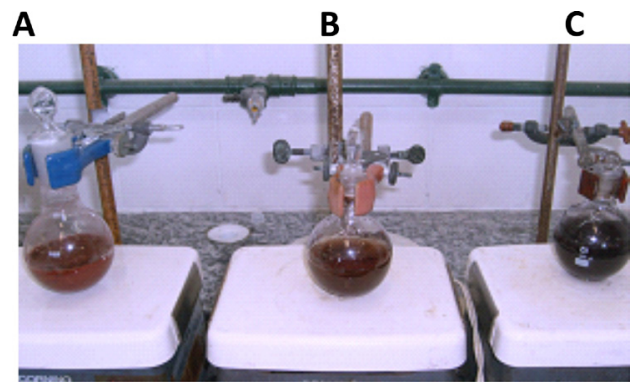

Figura 1. Reações da 1,4-naftoquinona com anilina $(\boldsymbol{A})$, p-toluidina $(\boldsymbol{B}) e$ p-anisidina $(\boldsymbol{C})$

Observou-se por CCD a formação de traços de um produto mais polar, que pode ser devido à adição da anilina à carbonila formando imino-naftoquinonas (Figura 16S, material suplementar). A 1,4-naftoquinona é um exemplo de substância $\alpha, \beta$-insaturada e devido a isso tem comportamento ambidentado quando reage com nucleófilos, podendo sofrer adição do tipo 1,4 (ataque ao carbono $\beta$ da olefina) ou adição direta à carbonila (Esquema 2). A posição preferencial da adição nucleofílica vai depender da natureza da anilina e das condições da reação. ${ }^{20}$

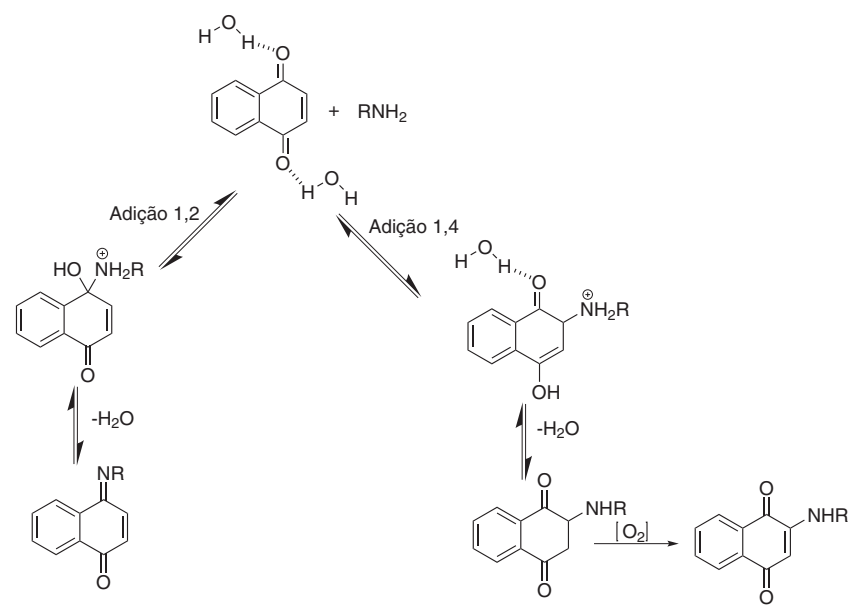

Esquema 2. Caminhos para formação de produtos via adição 1,4 e 1,2

A teoria de Pearson, que classifica os ácidos e bases em duros e moles, explica porque certas substâncias têm maior afinidade pela olefina, enquanto outras têm afinidade pelo carbono da carbonila. Segundo esta teoria, bases duras têm afinidade por ácido duros e bases moles por ácidos moles. ${ }^{21}$ Anilinas são bases mais moles que aminas alifáticas, porque o par de elétrons do átomo de nitrogênio interage com os orbitais p dos átomos de carbono do anel aromático.

No entanto, dependendo da natureza do substituinte presente no anel aromático, as anilinas podem apresentar diferentes graus de moleza. Com substituintes retiradores de elétrons, a deslocalização do par de elétrons do nitrogênio é maior devido à diminuição da densidade eletrônica do anel aromático e, consequentemente, a moleza.
O oposto é verificado quando o anel possui substituintes doadores de elétrons. A p-metóxi-anilina e a $p$-metil-anilina, que são bases mais duras do que a anilina, além de promoverem a adição 1,4 (produto majoritário), podem levar à formação minoritária de produtos resultantes da adição à carbonila.

A reação de adição de hidrazinas, hidroxilaminas e anilinas a compostos carbonilados é reversível e suas taxas de conversão são fortemente influenciadas pela acidez do meio de reação. Diversos estudos variando o pH e concentração são descritos na literatura. Em geral, a maior taxa de conversão é obtida em $\mathrm{pH}$ por volta de $4 .{ }^{22}$

Em virtude da alta reatividade da 1,4-naftoquinona, as reações mostradas neste artigo ocorrem em água sem a adição de catalisador.

Os experimentos foram repetidos por 8 estudantes do curso noturno de Licenciatura em Química da UFRJ. Os estudantes acompanharam a evolução das reações por CCD, comparando o $\mathrm{R}_{\mathrm{f}}$ dos produtos com os materiais de partida. Como tanto a 1,4-naftoquinona - material de partida - como, provavelmente, o produto de adição à carbonila (não identificado e formado em pequena quantidade, Figura $16 \mathrm{~S}$, material suplementar) não têm cores tão intensas, os estudantes usaram a câmara de luz ultravioleta $(\lambda=254 \mathrm{~nm})$ para melhor visualização dessas substâncias.

A adição das anilinas à 1,4-naftoquinona também pode ser realizada em fase sólida, apenas macerando os reagentes com um pistilo em um almofariz. Estas reações foram feitas para mostrar aos estudantes que algumas reações podem ocorrer sem solvente. Neste caso, as reações foram qualitativas e grande parte do material de partida não foi consumido.

\section{CONCLUSÃO}

A reação de adição de aminas à 1,4-naftoquinona é um experimento simples e atrativo, pois é possível observar imediatamente a formação do produto. Trata-se de uma proposta que considera os princípios da Química Verde, aborda conceitos como Economia Atômica, teoria de ácido duros e moles, reações em água e em fase sólida. As reações são facilmente acompanhadas por CCD e podem ser feitas entre 3 e $4 \mathrm{~h}$, geralmente o tempo disponível para as aulas de Química Orgânica Experimental.

\section{MATERIAL SUPLEMENTAR}

Está disponível em http://quimicanova.sbq.org.br, na forma de arquivo pdf e com acesso livre.

\section{PARTE EXPERIMENTAL}

\section{Materiais e métodos}

A caracterização dos produtos obtidos já está descrita na literatura. ${ }^{5}$ A ressonância magnética nuclear $(\mathrm{RMN})$ de hidrogênio $\left({ }^{1} \mathrm{H}\right) \mathrm{e}$ de carbono $\left({ }^{13} \mathrm{C}\right)$ e ultravioleta (ver material suplementar), utilizadas neste trabalho, serviram apenas para confirmação das estruturas.

Os espectros de $\mathrm{RMN}$ de ${ }^{1} \mathrm{H}$ e ${ }^{13} \mathrm{C}$ foram obtidos em aparelho Bruker DRX em frequências de 200 e $50 \mathrm{MHz}$, respectivamente. Foram utilizados como solventes uma mistura de clorofórmio $\left(\mathrm{CDCl}_{3}\right)$ e dimetilsulfóxido (DMSO-d $\mathrm{d}_{6}$ ).

As medidas de absorbância foram obtidas empregando-se espectrofotômetro UV-visível Varian modelo Cary 1E, utilizando cela de $1 \mathrm{~cm}$ de caminho óptico. As concentrações das soluções, preparadas em metanol, foram de $10^{-4} \mathrm{M}$.

As análises por CGAR-EM foram realizadas em cromatógrafo Agilent modelo 6850 acoplado a um detetor de espectrometria de massas Agilent modelo 5975C com ionização por impacto de elétrons 
a $70 \mathrm{eV}$ e analisador do tipo quadrupolo. A coluna capilar utilizada foi DB-1 ( $\&$ W) $30 \mathrm{~m}$ x 0,25 mm x 0,10 $\mu \mathrm{m}$ de espessura de fase. $\mathrm{O}$ gás de arraste utilizado foi hélio $\left(1,7 \mathrm{~mL} \mathrm{~min}^{-1}\right)$ e a pressão na cabeça da coluna foi de 8 psi. O volume de injeção da amostra foi $1,0 \mu \mathrm{L}$ com divisão de fluxo de 1:20. A temperatura inicial do forno foi de $100{ }^{\circ} \mathrm{C}$, mantida por $5 \mathrm{~min}$, com taxa de de $13{ }^{\circ} \mathrm{C} \mathrm{min}^{-1}$ até $270{ }^{\circ} \mathrm{C}$, permanecendo nesta temperatura por $20 \mathrm{~min}$. $\mathrm{O}$ tempo total de corrida foi $38,08 \mathrm{~min}$. O injetor foi operado a $290{ }^{\circ} \mathrm{C}$ e a interface a $300{ }^{\circ} \mathrm{C}$.

\section{Método geral para reação entre a 1,4-naftoquinona e anilinas}

\section{Reação empregando água como solvente}

Em um balão de $125 \mathrm{~mL}$ foram adicionados $50 \mathrm{~mL}$ de água e 3,5 mmol da anilina, $p$-metil-anilina ou p-metóxi-anilina. $\mathrm{O}$ meio de reação foi submetido à agitação vigorosa e, em seguida, foram lentamente adicionados 3,2 mmol de 1,4-naftoquinona. A coloração da suspensão tornou-se cada vez mais intensa com a adição da naftoquinona até adquirir uma cor vermelha escura nas reações com anilina e $p$-toluidina, e roxa, com a $p$-anisidina. A reação permaneceu sob agitação magnética durante $2 \mathrm{~h}$ e, neste período, a sua evolução foi acompanhada por CCD. Nos três casos os produtos foram filtrados sob pressão reduzida e lavados com água gelada.

\section{Reação sem solvente}

Em um almofariz foram adicionados $0,158 \mathrm{~g}(1 \mathrm{mmol}) \mathrm{de}$ 1,4-naftoquinona e 1,3 mmol da anilina, $p$-metil-anilina ou $p$-metóxianilina. A mistura foi macerada com um pistilo até homogeneização do sistema, em seguida, dissolvida em $12 \mathrm{~mL}$ de diclorometano e a fase orgânica lavada com solução aquosa de ácido clorídrico $10 \%$ $(10 \mathrm{~mL})$ e, posteriormente, com água $(2 \times 10 \mathrm{~mL})$. A fase orgânica foi secada com sulfato de sódio anidro, filtrada e concentrada à secura, sob pressão reduzida, em evaporador rotatório.

\section{REFERÊNCIAS}

1. Freire, C. P. V.; Ferreira, S. B.; de Oliveira, N. S. M.; Matsuura, A. B. J.; Gama, I. L.; da Silva, F. C.; de Souza, M. C. B. V.; Lima, E. S.; Ferreira, V. F.; MedChemComm. 2010, 1, 229.

2. da Silva-Jr, E. N.; de Deus, C. F.; Cavalcanti, B. C.; Pessoa, C.; CostaLotufo, L. V.; Montenegro, R. C.; de Moraes, M. O.; Pinto, M. C. F. R.; de Simone, C. A.; Ferreira, V. F.; Goulart, M. O. F.; Andrade, C. K. Z.; Pinto, A. V.; J. Med. Chem. 2010, 53, 504; da Rocha, D. R.; de Souza, A. C. G.; Resende, J. A. L. C.; Santos, W. C.; dos Santos, E. A.; Pessoa, C.; de Moraes, M. O.; Costa-Lotufo, L. V. ; Montenegro, R. C.; Ferreira, V. F.; Org. Biomol. Chem. 2011, 9, 4315; Francisco, A. I.; Vargas, M. D.; Fragoso, T. P.; Carneiro, J. W. M.; Casellato, A.; da Silva, F. C.; Ferreira, V. F.; Barbosa, J. P; Pessoa, C.; Costa-Lotufo, L. V.; Marinho Filho, J. D. B.; de Moraes, M. O.; Mangrich, A. S.; J. Braz. Chem. Soc. 2010, 21, 1293; Silva-Jr, E. N.; de Moura, M. A. B. F.; Pinto, A. V.; Pinto, M. C. F. R.; Souza, M. C. B. V.; Araújo, A. J.; Pessoa, C.; Costa-Lotufo, L. V.; Montenegro, R. C.; Moraes, M. O.; Ferreira, V. F.; Goulart, M. O. F.; J. Braz. Chem. Soc. 2009, 20, 635.

3. Lourenço, A. L.; Abreu, P. A.; Leal, B.; Silva-Jr, E. N.; Pinto, A. V.; Pinto, M. C. F. R.; Souza, A. M. T.; Novais, J. S.; Paiva, M. B.; Cabral, L. M.; Rodrigues, C. R.; Ferreira, V. F.; Castro, H. C.; Curr. Microbiol. 2011, 62, 684.

4. Sacau, E. P.; Braun, A. E.; Ravelo, A. G.; Yapu, D. G.; Turba, A. G.; Chem. Biodiversity 2005, 2, 264.

5. Ferreira, S. B.; Salomão, K.; da Silva, F. C.; Pinto, A. V.; Kaiser, C. R.; Pinto, A. C.; Ferreira, V. F.; de Castro, S. L.; Eur. J. Med. Chem. 2011, 46, 3071; Bourguignon, S. C.; Cavalcanti, D. F. B.; de Souza, A. M. T.; Castro, H. C.; Rodrigues, C. R.; Albuquerque, M. G.; Santos, D. O.; da Silva, G. G.; da Silva, F. C.; Ferreira, V. F.; de Pinho, R. T.; Alves, C. R.;
Experimental Parasitol. 2011, 127, 160; Bourguignon, S. C.; Castro, H. C.; Santos, D. O.; Alves, C. R.; Ferreira,V. F.; Gama, I. L.; Silva, F. C.; Seguins, W. S.; Pinho, R. T.; Experimental Parasitol. 2009, 122, 91.

6. Ferreira, S. B.; da Silva, F. C.; Bezerra, F. A. F. M.; Lourenço, M. C. S.; Kaiser, C. R.; Pinto, A. C.; Ferreira, V. F.; Archiv der Pharmazie 2010, $343,81$.

7. Matés, J. M.; Sánchez-Jiménez, F. M.; Int. J. Biochem. Cell Biol. 2000, 32, 157.

8. Silva, T. M. S.; Camara, C. A.; Barbosa, T. P.; Soares, A. Z.; Cunha, L. C.; Pinto, A. C.; Vargas, M. D.; Bioorg. Med. Chem. 2005, 13, 193.

9. Oliveira, C. G. T.; Miranda, F. F.; Ferreira, V. F.; Freitas, C. C.; Rabello, R. F.; Carballido, J. M.; Corrêa, L. C. D.; J. Braz. Chem. Soc. 2001, 12, 339; Fieser, L. F.; Hartwell, J. L.; J. Am. Chem. Soc. 1935, 57, 1482.

10. Francisco, A. I.; Casellato, A.; Neves, A. P.; Carneiro, J. W. M.; Vargas, M. D.; Visentin, L. C.; Magalhães, A.; Câmara, C. A.; Pessoa, C.; CostaLotufo, L. V.; Marinho Filho, J. D. B.; Moraes, M. O.; J. Braz. Chem. Soc. 2010, 21, 169; Camara, C. A.; Pinto, A. C.; Rosa, M. A.; Vargas, M. D.; Tetrahedron 2001, 57, 9569; Cunha, A. S.; Lima, E. L. S.; Pinto, A. C.; Esteves-Souza, A.; Echevarria, A.; Camara, C. A.; Vargas, M. D.; Torres, J. C.; J. Braz. Chem. Soc. 2006, 17, 439; Francisco, A. I.; Vargas, M. D.; Carneiro, J. W. M.; Lanznaster, M.; Torres, J. C.; Camara, C. A.; Pinto, A. C.; J. Mol. Struct. 2008, 891, 228.

11. Tapia, R. A.; Cantuarias, L.; Cuéllar, M.; Villena, J.; J. Braz. Chem. Soc. 2009, 20, 999.

12. Franco, C. F. J.; Jordão, A. K.; Ferreira, V. F.; Pinto, A. C.; Souza, M. C. B. V.; Resende, J. A. L. C.; Cunha, A. C.; J. Braz. Chem. Soc. 2011, 22, 187.

13. Cunha, S.; Santos, L. F. P.; Rocha, Z. N.; Rivelino, R.; Ferrari, J.; Vencato, I.; Lariucci, C.; Quim. Nova 2010, 33, 2108.

14. Valderrama, J. A.; Ibacache, J. A.; Arancibia, V.; Rodriguez, J.; Theoduloz, C.; Bioorg. Med. Chem. 2009, 17, 2894.

15. Wu, X.; Larhed, M.; Org. Lett. 2005, 7, 3327; Wijnen, J. W.; Engberts, J. B. F. N.; J. Org. Chem. 1997, 62, 2039; Yamada, Y. M. A.; Takeda, K.; Takahashi, H.; Ikegami, S.; J. Org. Chem. 2003, 68, 7733; Jones Jr., J.; Silva, F. M.; Almeida, Q. A. R.; Coelho, R. B.; Gonçalves, M.; Ferre, F. T.; Sena, J. D.; Heterocycl. Commun. 2009, 15, 57; da Silva, F. C.; Res. J. Chem. Environ. 2007, 11, 72.

16. Cao, Y.- J.; Lai, Y. -Y.; Wang, X.; Li, Y.- J.; Xiao, W. -J.; Tetrahedron Lett. 2007, 48, 21; Ko, K.; Nakano, K.; Watanabe, S.; Ichikawa, Y.; Kotsuki, H.; Tetrahedron Lett. 2009, 50, 4025; Chandrasekhar, S.; Kumar, T. P.; Haribabu, K.; Reddy, C. R.; Tetrahedron: Asymmetry 2010, 21, 2372; Da Silva, F. M.; Almeida, Q. A. R.; Pereira, M. L. O.; Coelho, R. B.; Carvalho, E. M.; Kaiser, C. R.; Jones Jr, J.; Da Silva, F. M.; J. Braz. Chem. Soc. 2008, 19, 894.

17. Lenardão, E. J.; Freitag, R. A.; Dabdoub, M. J.; Batista, A. C. F.; Silveira, C. C.; Quim. Nova 2003, 26, 123; Da Silva, F. M.; Lacerda, P. S. B.; Jones Jr., J.; Quim. Nova 2005, 28, 103; Uozumi, Y.; Synlett 2010, 1988.

18. Teixeira, E. F.; Santos, A. P. B.; Bastos, R. S.; Pinto, A. C.; Kümmerle, A. E.; Coelho, R. R.; Quim. Nova 2010, 33, 1603; Silva, B. N. M.; Bastos, R. S.; Silva, B. V.; Pinto, A. C.; Quim. Nova 2010, 33, 2279; Santos, A. P. B.; Gonçalves, I. R. C.; Pais, K. C.; Martinez, S. T.; Lachter, E. R.; Pinto, A. C.; Quim. Nova 2009, 32, 1667; Silva, R. B.; Torres, J. C.; Garden, S. J.; Violante, F. A.; Rezende, M. J. C.; Silva, B. V.; Pinto, A. C.; Quim. Nova 2008, 31, 924; Bastos, R. S.; Cunha, A. S.; Silva, L. C.; Oliveira, C. C. P.; Rezende, C. M.; Pinto, A. C.; Quim. Nova 2008, 31, 172.

19. Lisboa, C. S.; Santos, V. G.; Vaz, B. G.; Lucas, N. C.; Eberlin, M. N.; Garden, S. J.; J. Org. Chem. 2011, 76, 5264.

20. Costa, P.; Pilli, R.; Pinheiro, S.; Vasconcellos, M.; Substâncias carboniladas e derivados, $1^{\mathrm{a}}$ ed., Bookman: Porto Alegre, 2003.

21. Costa, P.; Ferreira, V.; Esteves, P.; Vasconcellos, M.; Ácidos e Bases em Química Orgânica, $1^{\text {a }}$ ed., Bookman: Porto Alegre, 2005; Arroio, A.; Honório, K. M.; da Silva, A. B. F.; Quim. Nova 2010, 33, 694.

22. Conant, J. B.; Barlett, P. D.; J. Am. Chem. Soc. 1932, 54, 2881. 